\title{
UN EJEMPLO DEL VALOR AGREGADO QUE APORTAN LAS TIC EN LA EDUCACIÓN UNIVERSITARIA
}

CARMEN RESCALA GRICELA ROHDE

RITA PAVÓN

FACULTAD DE CIENCIAS ECONÓMICAS - UNNE 


\title{
RESUMEN
}

\begin{abstract}
1 mundo actual, con cambios permanentes que con llevan adelantos científicos y tecnológicos, nos obliga a delinear nuevas estrategias educativas para mejorar el proceso de enseñanza-aprendizaje, brindando a nuestros alumnos las herramientas informáticas que les permitirán plantear y resolver modelos que representen situaciones reales, transformando la realidad económica en la búsqueda de la optimización de la labor que desarrollen en el mundo de los negocios.

$\mathrm{Al}$ enseñar contenidos de los programas de las distintas asignaturas del área matemática, las TIC (tecnologías de la información y de las comunicaciones) constituyen un recurso didáctico valioso, potente, que favorece el estudio independiente y eficiente para alcanzar los objetivos que nos planteamos en las planificaciones de cada curso de Matemática.

El objetivo de este trabajo es mostrar al lector como el uso de las TIC en la educación universitaria genera un valor agregado que beneficia al proceso de enseñanza- aprendizaje. Mediante la descripción de un ejemplo de la unidad de programación lineal se permite apreciar las formas en que las herramientas informáticas aportan un valor (al que llamamos valor agregado) al suceso de elaborar y asimilar conocimientos.
\end{abstract}

Palabras clave: Matemática, Informática, Proceso Educativo, Solver, Graph.

\begin{abstract}
The current world, with permanent changes, that lead scientifics and technologic advances, forces us to delineate new educative strategies to improve the teaching-learning process, providing our students the computer science tools that'll let them formulate and solve models that represent real situations transforming the economic reality that they face, seeking to optimize the work developed in the business world.

In the teaching of the contents of the programs of the different subjects of the mathematical area, the TICs (information and communications technology) constitute a didactic, valuable, powerful resource, which favors the independent and efficient study to reach the aims that we posed in every course of Mathematics. The objective of this paper, is to show to the reader how the use of ICT in higher education generates an added value that benefits the teachinglearning process by describing an example of linear programming unit to appreciate the ways in which the informatics tools provides a value (which we call added value) to the fact of the development and assimilation of knowledge..
\end{abstract}

Keywords: mathematics, computer science, educational process, solver, graph 


\section{INTRODUCCIÓN}

La enseñanza de los contenidos matemáticos a impartir debe ayudar a nuestros estudiantes a entender, apreciar y realizar un análisis matemático aplicado a partir del análisis matemático puro. En ese quehacer de la matemática aplicada a cuestiones referidas a las áreas de la administración, la economía y la gestión de negocios, tiene una fundamental importancia la utilización de herramientas informáticas.

Las herramientas que suministra la informática al desarrollo de los contenidos matemáticos de las diferentes asignaturas del área son instrumentos que sinergizan la evolución del proceso enseñanza-aprendizaje y aportan el valor agregado que lo jerarquiza.

Para su desarrollo integral, el futuro profesional necesita de conocimientos que deben estar presentes en su formación, caso contrario le será muy difícil insertarse en una sociedad demandante de servicios realizados con calidad y experiencia. Esta necesidad comienza con la de los docentes de capacitarse introduciéndose en el manejo de cuestiones tecnológicas.

Los conocimientos matemáticos producen cambios y avances permanentes en innovaciones y desarrollo, haciendo de las transformaciones una rutina para el progreso del mundo y la satisfacción de las necesidades del hombre. A estos conocimientos debemos sumarle los aportes de la informática y afirmar que ambos se retroalimentan, contribuyendo al bienestar de la humanidad y al avance de las ciencias. Matemática e Informática, operan como una biyectividad, la informática como herramienta para la ciencia matemática no hubiera podido desarrollarse sin los conceptos que ésta aportó para el desenvolvimiento de los programas que hoy nos auxilian en el trabajo diario y la Matemática no podría avanzar sin el auxilio de la Informática que agrega un valor indispensable en la enseñanza de la misma.

Si consideramos el proceso educativo, puede afirmarse que es muy reciente la formación de los docentes universitarios en el manejo de programas informáticos que colaboran con la tarea que desarrollan. Con anterioridad, la enseñanza de la matemática en los cursos de Facultades de Ciencias Económicas se veía privada de realizar análisis que involucraran varias variables de decisión o desarrollos de trabajos más complejos de los que venían realizándose. Les correspondía a nuestros egresados desarrollar, con el auxilio de expertos en informática, sus propios programas para cumplir con la exigencia de sus clientes y así poder delinear estrategias que resolvieran situaciones reales.

Gracias al desarrollo tecnológico de los últimos años y a la posibilidad de las computadoras personales, tanto los profesionales como los docentes capaces de insertarse en ese ámbito tecnológico, pudieron hacer uso de las poderosas herramientas que son los diferentes programas de computadoras, provocándose desde entonces un importante avance en el proceso educativo.

Cuando en las carreras universitarias de las Facultades de Ciencias Económicas enseñamos los contenidos de los programas de los planes de estudio, orientamos a nuestros alumnos para que resuelvan los ejercicios que se proponen utilizando el correspondiente software, el que al ser aplicado, produce economía de tiempos y evita errores de cálculo. 
Hoy, es común el uso de software en los ámbitos educativos, teniendo relevancia en la esfera universitaria por involucrar tanto a las actividades administrativas como a las académicas y a las de investigación.

Al enseñar contenidos matemáticos a través del uso de herramientas informáticas convertimos al alumno en partícipe activo del proceso educativo, por lo tanto debemos conducirlos a que primero planifiquen lo que van a desarrollar para luego crear el conjunto de variables, obtener resultados a través de iteraciones y analizar esos resultados teniendo presente que el que seleccionen deberá satisfacer a la situación real para la cual se efectuó la investigación.

En el programa de la asignatura MATEMÁTICA I de la Facultad de Ciencias Económicas de la UNNE, en la unidad 7 se trata el modelo de Programación Lineal, recomendamos al impartir los conceptos de este modelo el uso de software que permitan la representación gráfica y el hallar los valores óptimos del problema que se quiera resolver. Es por esto que podemos afirmar que en la actualidad la enseñanza de los temas del programa de esta materia tiene un valor agregado dado por la herramienta informática.

Existen factores que nos imponen ir al encuentro de soluciones factibles para implementar la enseñanza de algunos contenidos de matemática a través de la informática, esos factores son: los presupuestos universitarios que no alcanzan para contemplar todas las necesidades de equipamientos duros y blandos (creación de gabinetes de informática en los cuales trabajar con la gran cantidad de alumnos que cursan las distintas carreras de las facultades y paquetes de software) y que muchos alumnos carecen de recursos. Esas soluciones factibles consisten en utilizar, en particular, para la enseñanza de contenidos de Matemática I de la Facultad de Ciencias Económicas de la UNNE, la Planilla de cálculo de EXCEL y el software GRAPH, ambos de fácil instalación y comprensión.

La herramienta SOLVER de Excel, por formar parte del paquete de Microsoft Office está al alcance de cualquier estudiante, de cualquier docente y es, para todos aquellos que deseen hacer representaciones gráficas, iteraciones o aplicar algoritmos, fácil de usar, ya sea en el ámbito educativo como en el profesional y en el personal.

El software GRAPH es de descarga gratuita, de poco peso, de sencilla instalación y desinstalación, tiene una interfase de fácil comprensión y utilización, compatible con Windows. En la página oficial se puede descargar el manual en forma gratuita. Los datos para las representaciones gráficas pueden operarse de manera ágil y a continuación guardar los escenarios y los gráficos en forma dinámica, separada, accesible de regresar a ellos en el momento en que se los requiere.

En párrafos anteriores consideramos el concepto de valor agregado para el aporte de la herramienta informática en el proceso de enseñanza-aprendizaje de contenidos matemáticos, pero, ¿̇cuáles son los ingredientes de ese valor agregado?

\footnotetext{
${ }^{1}$ Se destaca que la información contenida en el presente acápite fue obtenida mediante un trabajo de campo en el cual se han recorrido sucursales ubicadas en la Ciudad de Corrientes -Provincia de Corrientes, Argentina- de diferentes entidades bancarias (Banco Galicia, Banco Francés, Banco Santander Río, Banco de Corrientes, Banco Nación, entre otros), grandes entidades financieras (Vgr. Tarjeta Naranja, Confina, Efectivo Sí, Credil, Credilogros), pequeñas empresas financieras (Vgr. Crédito Azteca, Dinero Ya, Metroshop, Rapi Cuotas, entre otros) y empresas otorgantes de crédito para la adquisición de bienes (Vgr. Red Megatone, Frávega, Garbarino y La Gran Palmira, entre otras).
} 
Primero creemos apropiado dar el concepto de valor agregado que conviene a este trabajo y decimos que: valor agregado es el que implica el mayor valor que una mercancía recibe al pasar por las distintas etapas del proceso productivo.

"El criterio del valor agregado se basa en el hecho de que a medida que un producto pasa de uno a otro sector se le va agregando valor hasta llegar a la etapa final en que su precio resulta de la causa de todos los valores agregados" (Besil, 2007).

Si queremos referir el criterio de valor agregado al terreno de la educación e ilustrarlo con un ejemplo, parafraseando lo antes mencionado, podemos decir que el proceso de enseñanza -aprendizaje es nuestro proceso productivo y entonces afirmamos que el mismo tiene un valor agregado dado por el uso de la herramienta informática en una de las etapas de ese proceso. El éxito de ese proceso de enseñanza- aprendizaje será el resultado de todos los valores agregados.

En este Trabajo brindamos como ejemplo de nuestro proceso de enseñanza aprendizaje el tratamiento de un modelo de programación lineal, tema que cae en el área de la Investigación de Operaciones, la que forma parte de la Ciencia Matemática.

¿Qué es la Investigación de operaciones?

Es la técnica que se ocupa de la toma de decisiones óptimas a través del modelado de sistemas.

¿Qué es un modelo?

Un modelo es la representación ideal de un sistema y de la forma en que este opera. Su objetivo es analizar el comportamiento del sistema o bien predecir su comportamiento futuro.

Obviamente, los modelos no son tan complejos como el sistema mismo, de tal manera que se hacen las suposiciones y restricciones necesarias para representar las porciones más relevantes del mismo. El diseño y la aplicación de modelos matemáticos han producido verdaderas revoluciones en el campo científico y en el tecnológico.

En las distintas disciplinas de las Ciencias Económicas, las variables pueden ser representadas por símbolos y sus propiedades enunciadas en lenguaje matemático, lo que significa que la ciencia matemática aporta a esta ciencia social las herramientas y técnicas para analizar las relaciones entre sus variables.

Durante mucho tiempo los gerentes fueron indiferentes a la construcción de modelos cuantitativos que les permitieran tomar decisiones más acertadas, seguían confiando en su intuición y en su experiencia. Si bien reconocían las ventajas que presentaba la construcción de modelos, opinaban que solamente podían crear modelos los matemáticos, los consultores y los expertos en informática.

Al delegar la construcción de modelos a los especialistas en el tema, el management o administrador general o gerente, no participaba del proceso de construcción de los modelos que ayudarían a tomar decisiones, por lo cual la interpretación de los resultados era errónea o no se llevaba a la práctica. Éste fue el motivo por el cual los gerentes aún reacios a la administración científica, afirmaban que los modelos sólo servían para que con los resultados se redactaran informes que nadie leía. 
Esto significaba pérdida de esfuerzos, dinero y tiempo, perjudicándose las organizaciones que los habían solicitado, ya que estos procesos de modelado no incidían en la gestión de administración de las empresas.

Sin embargo, sucede algo que revierte el pensamiento de esos gerentes de empresas.

Ese importante suceso es el avance tecnológico, gracias a él los administradores comienzan a construir y analizar sus propios modelos.

Ese avance tecnológico está dado por:

* el poder de las computadoras personales y

* los programas accesibles y fáciles de usar.

En la actualidad, la informática es una herramienta indispensable para desarrollar contenidos matemáticos, la característica de indispensable la adquiere porque sin ella estamos limitados en la resolución de muchos problemas matemáticos que implican modelos de procesos iterativos y numerosos cálculos.

¿Quiénes son los receptores directos del ejemplo que planteamos? ¿Cuáles son sus características?

En el proceso educativo es fundamental conocer las características del alumno al que va dirigida la enseñanza. Un alumno de Matemática I tiene alrededor de 18 años, según Córica y Dinerstein (2009), el acceso a la información y el manejo de las nuevas tecnologías les ha otorgado una sensación de poder y actitud retadora. Les interesa aprender lo máximo posible en el menor tiempo; más práctica y aplicaciones que teoría.

Los jóvenes de esta generación tienen más desarrollado el hemisferio derecho del cerebro, el creativo, a diferencia de las generaciones anteriores que desarrollaban más el izquierdo, el de la lectura de un libro y el de la lógica.

El acceso a internet los capacitó para clasificar la información en útil o inútil, en actual u obsoleta, esta mentalidad está presente en una clase, el alumno también clasificará los contenidos que recibe. Muchos programas educativos actuales siguen apuntando al hemisferio izquierdo, no es que leer un libro o seguir un desarrollo lógico pierda vigencia sino que será necesario estimular primero al hemisferio derecho para llegar después al izquierdo. Las TIC favorecen el estudio independiente como así también las actividades en otros tiempos: asincrónica (tiempo diferido) y sincrónica (tiempo actual).

Los integrantes de esta generación aceptan la diversidad en todos sus aspectos, un mundo sin fronteras donde la web tiene principal importancia, les cuesta entender las razones para reconocer la autoridad, prefieren la reciprocidad que obtienen utilizando la "red", por eso cuestionan a los adultos, no por indisciplina sino por el poder de acceso a la información que tienen.

Otras características de esta generación conocida como NET o WHY son la impaciencia y el impedimento de llevar a cabo tareas que no resulten satisfactorias inmediatamente.

Buscan las soluciones fáciles, las que no requieren mucho esfuerzo; es la educación la que puede construir en ellos la idea de proceso. Los jóvenes de esta generación se involucran cuan- 
do se les permite ser activos en su aprendizaje, pasando de la obligación al placer por aprender, de un aprendizaje lineal al aprendizaje con herramientas informáticas. (Ferreiro, 2000)

Todo cambio genera resistencia, no solamente en los alumnos sino en también en los docentes, son muchos los argumentos que se esgrimen, por ejemplo un docente puede afirmar que se pierde autoridad, se genera caos, indisciplina en un gabinete de computación, pero estas afirmaciones solo demuestran miedos, miedo a perder la autoridad, porque ya no se está frente al curso sino que actúa como mediador, como facilitador del aprendizaje a través de las nuevas tecnologías, adoptando un paradigma centrado en el estudiante en lugar de uno centrado en el docente.

\section{EL PROBLEMA DE LA PRODUCCIÓN DE PELOTAS}

La empresa Wilson manufactura dos tipos de pelotas: de fútbol 5 y de vóley. La fábrica tiene 900 horas disponibles de tiempo de producción en su departamento corte y costura, 270 horas disponibles en el departamento terminado y 140 horas disponibles en el departamento empaque y expedición. Los requerimientos de tiempo de producción, en horas, para las pelotas de futbol 5 son: corte y costura 1 hs, terminado $1 / 2$ hs, empaque y expedición $1 / 10$ hs. Los requerimientos de tiempo de producción en horas para las pelotas de vóley son: corte y costura $11 / 2 \mathrm{hs}$, terminado $3 / 10 \mathrm{hs}$ y empaque y expedición $1 / 4 \mathrm{hs}$. Las ganancias que reportan las ventas por unidad de cada tipo de pelota son: $\$ 5$ para las pelotas de futbol 5 y $\$ 8$ para las pelotas de vóley. Los datos fueron volcados en la siguiente tabla:

\begin{tabular}{|l|c|c|c|c|}
\hline & \multicolumn{3}{|c|}{ Tiempos de producción en horas } & \\
\hline & Corte y costura & Terminado & $\begin{array}{l}\text { Empaque y } \\
\text { expedición }\end{array}$ & $\begin{array}{c}\text { Ganancia por } \\
\text { unidad vendida }\end{array}$ \\
\hline Pelotas-fútbol & 1 & $1 / 2$ & $1 / 10$ & $\$ 5$ \\
\hline Pelotas voley & $3 / 2$ & $3 / 10$ & $1 / 4$ & $\$ 8$ \\
\hline Restricciones & 900 & 270 & 140 & \\
\hline
\end{tabular}

Tabla 1. Datos del problema

Teniendo en cuenta que la empresa está interesada en maximizar las ganancias totales, debemos responder:

1-¿Cuál es el modelo de programación lineal ${ }^{1}$ para este problema?

2-¿A cuánto asciende el máximo beneficio que se puede obtener dado los recursos disponibles?

3-¿Cuántas pelotas tiene que fabricar la empresa Wilson para maximizar sus ganancias?

4-¿Cuántas horas de trabajo se deben programar en el departamento "Empaque y Expedición"?

\footnotetext{
${ }^{1}$ Programación lineal: técnica matemática de optimización; optimizar es maximizar o minimizar un objetivo.
} 
5-¿Cuál es el tiempo de holgura² en los departamentos?

6-Encontrar la solución óptima usando el procedimiento de resolución gráfica y verifique si las ganancias totales obtenidas con la herramienta Solver es la misma que se obtiene con el método gráfico.

7-Según el método gráfico, ¿Cuál es la restricción que no limita la región factible ${ }^{3}$ en el punto de la solución óptima?

Para empezar se formula el problema teniendo en cuenta:

* Función objetivo: En este caso, lo que buscamos es maximizar los beneficios totales por la venta de las pelotas.

* Variables de decisión: Son las variables de entradas que pueden controlarse en un modelo de programación lineal. Por ese motivo se llaman variables de decisión.

En nuestro ejemplo:

x: número de pelotas de fútbol a producir

y: número de pelotas de vóley a producir

* Restricciones estructurales: Estas restricciones son inecuaciones que representan una desigualdad que en el primer miembro tiene una sumatoria de términos en la cual, cada término es el producto del recurso que se utiliza por pelota por la cantidad de pelotas producidas y en el segundo miembro figura la cantidad del recurso de que dispone la empresa para la fabricación de las pelotas.

Determinadas las variables y con los datos extraídos de la tabla, nuestro modelo es el siguiente:

\section{Maximizar la función objetivo:}

$$
z=5^{x+8 y}
$$

Sujeto a las restricciones:

$$
\text { Estructurales }\left\{\begin{array}{c}
x+\frac{3}{2} y \leq 900 \\
\frac{1}{2} x+\frac{3}{10} y \leq 270 \\
\frac{1}{10} x+\frac{1}{4} y \leq 140 \\
x ; y \geq 0
\end{array}\right.
$$

\section{SOLUCIÓN GRÁFICA DEL PROBLEMA: GRAFICACIÓN EN GRAPH}

Definido el modelo del problema se trazan las rectas asociadas a las restricciones y para mostrar todas las soluciones que satisfacen las mismas, se representa la solución de cada una de las restricciones:

\footnotetext{
${ }^{2}$ Holgura: Indica las cantidades de los recursos no utilizados en el plan óptimo.

${ }^{3}$ Región factible: Todos los puntos ubicados en el área sombreada del gráfico que satisfacen simultáneamente a 2 o más restricciones.
} 


\begin{tabular}{|l|c|c|c|}
\hline & 1a Restricción & 2a Restricción & 3a Restricción \\
\hline Ecuación asociada a & $x+\frac{3}{2} y \leq 900$ & $\frac{1}{2} x+\frac{3}{10} y \leq 270$ & $\frac{1}{10} x+\frac{1}{4} y \leq 140$ \\
\hline Forma explícita & $y=600-\frac{2}{3} x$ & $y=900-\frac{5}{3} x$ & $y=560-\frac{2}{5} x$ \\
\hline
\end{tabular}

Tabla 2. Ecuaciones

1.1) En la barra de menú se elige Función-Insertar función

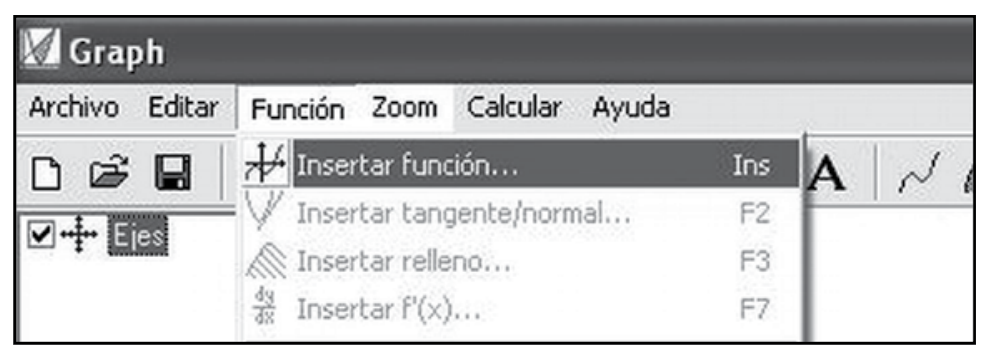

Gráfico 1. Función-Insertar función

En la ventana que aparece ingresamos la función en su forma explícita, sí en ella existen fracciones, éstas deben escribirse dentro de paréntesis.

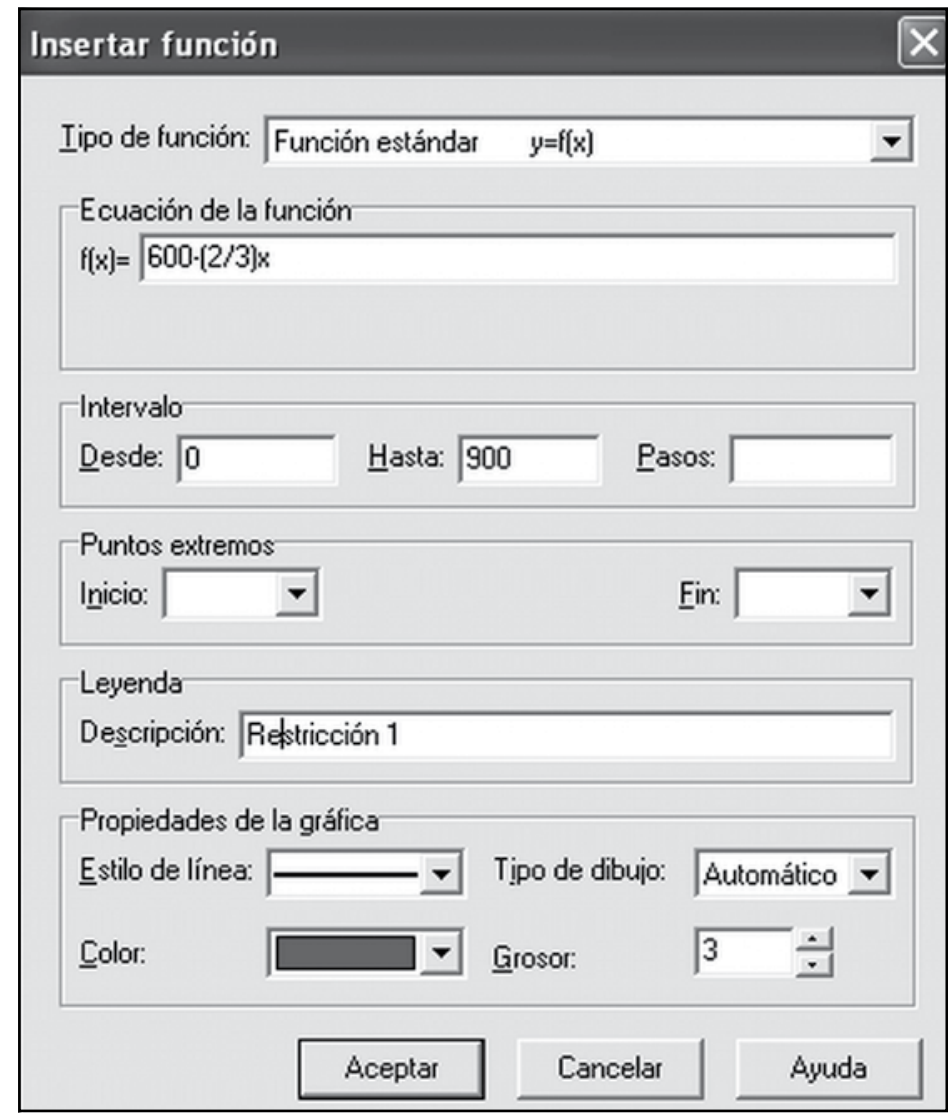

Gráfico 2. Insertar función 
Para respetar las restricciones de no negatividad, la representación de las rectas se convierte en la representación de segmentos en el primer cuadrante. El intervalo correcto para la primera recta es desde o hasta 900, asignamos nombre a la función para poder identificarla y luego procedemos a elegir el estilo de línea, color, grosor, finalmente damos aceptar.

A través del mismo procedimiento se grafican las rectas restantes y el resultado se puede ver en el siguiente gráfico:

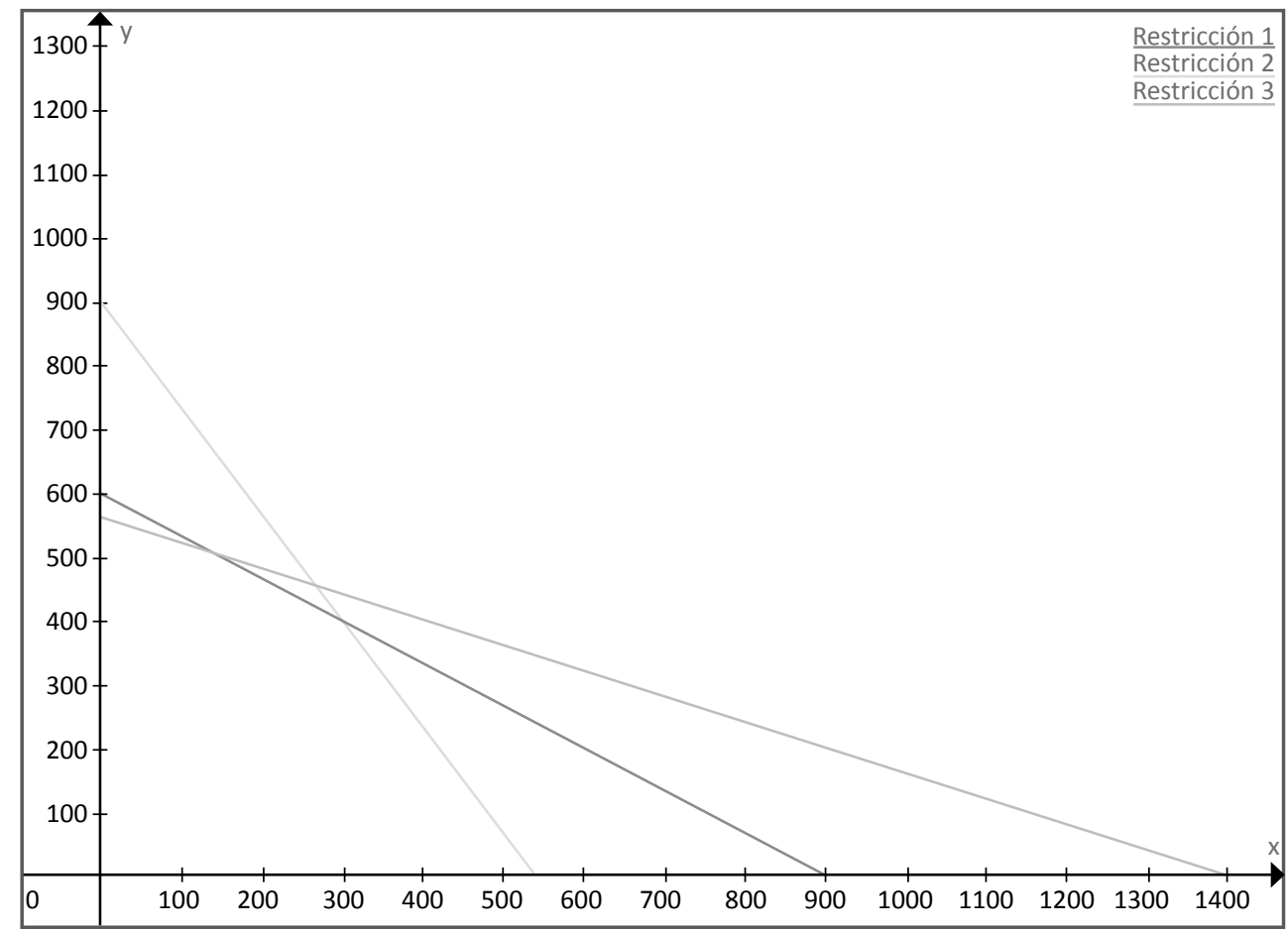

Gráfico 3. Resultado de la graficación

1.2) Para sombrear el área de soluciones factibles ${ }^{4}$ seleccionamos cualquiera de las restricciones, por ejemplo, la primera restricción.

* En la barra de menú se elige Función-Insertar relleno

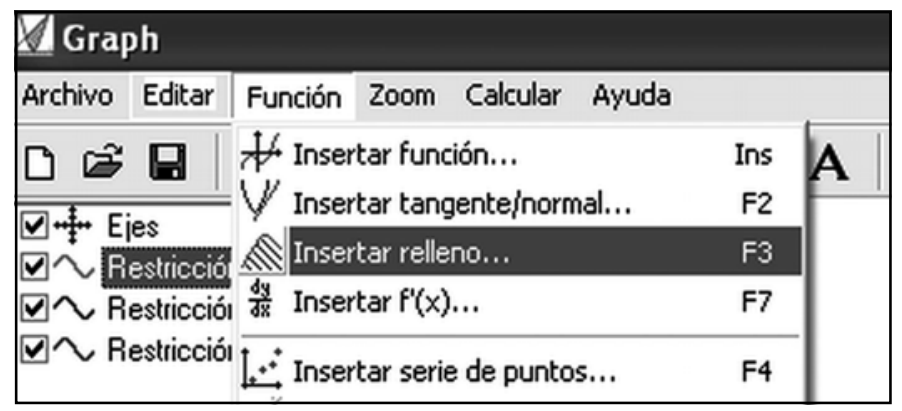

Gráfico 4. Función-Insertar relleno

\footnotetext{
${ }^{4}$ Área de soluciones factibles: Es el conjunto solución del sistema de restricciones que incluye todas las combinaciones de las variables de decisión que satisfacen las restricciones estructurales y de no negatividad. Esas combinaciones se consideran candidatas de ser la solución óptima.
} 
* En las opciones elegimos: Relleno: Entre la función y el eje x y en Opciones: intervalos desde o a aumentar hasta intersección.

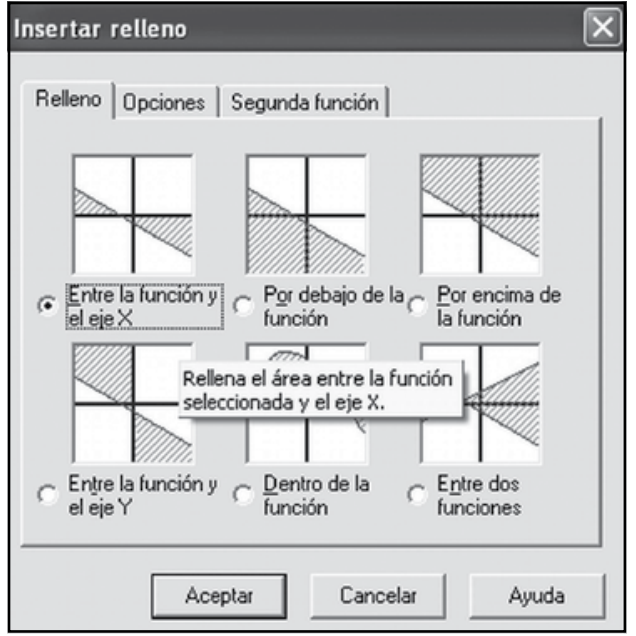

Gráfico 5. Insertar relleno-Relleno

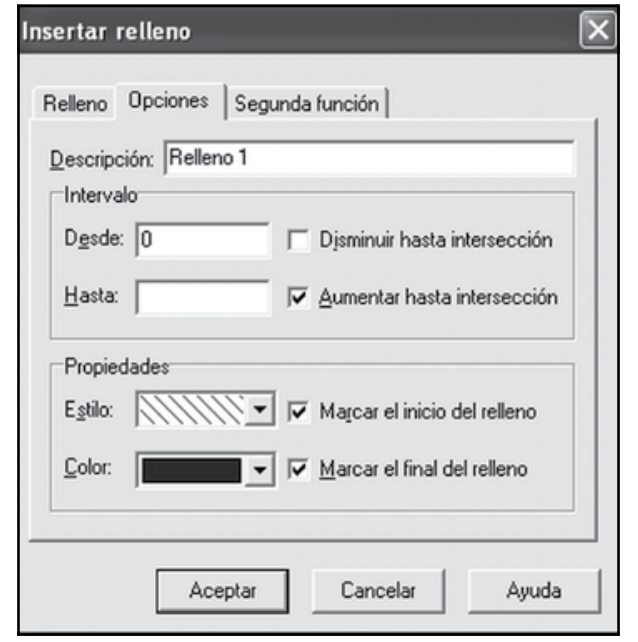

Gráfico 6. Insertar relleno-Opciones

* Seguimos los mismos pasos con las restantes restricciones.

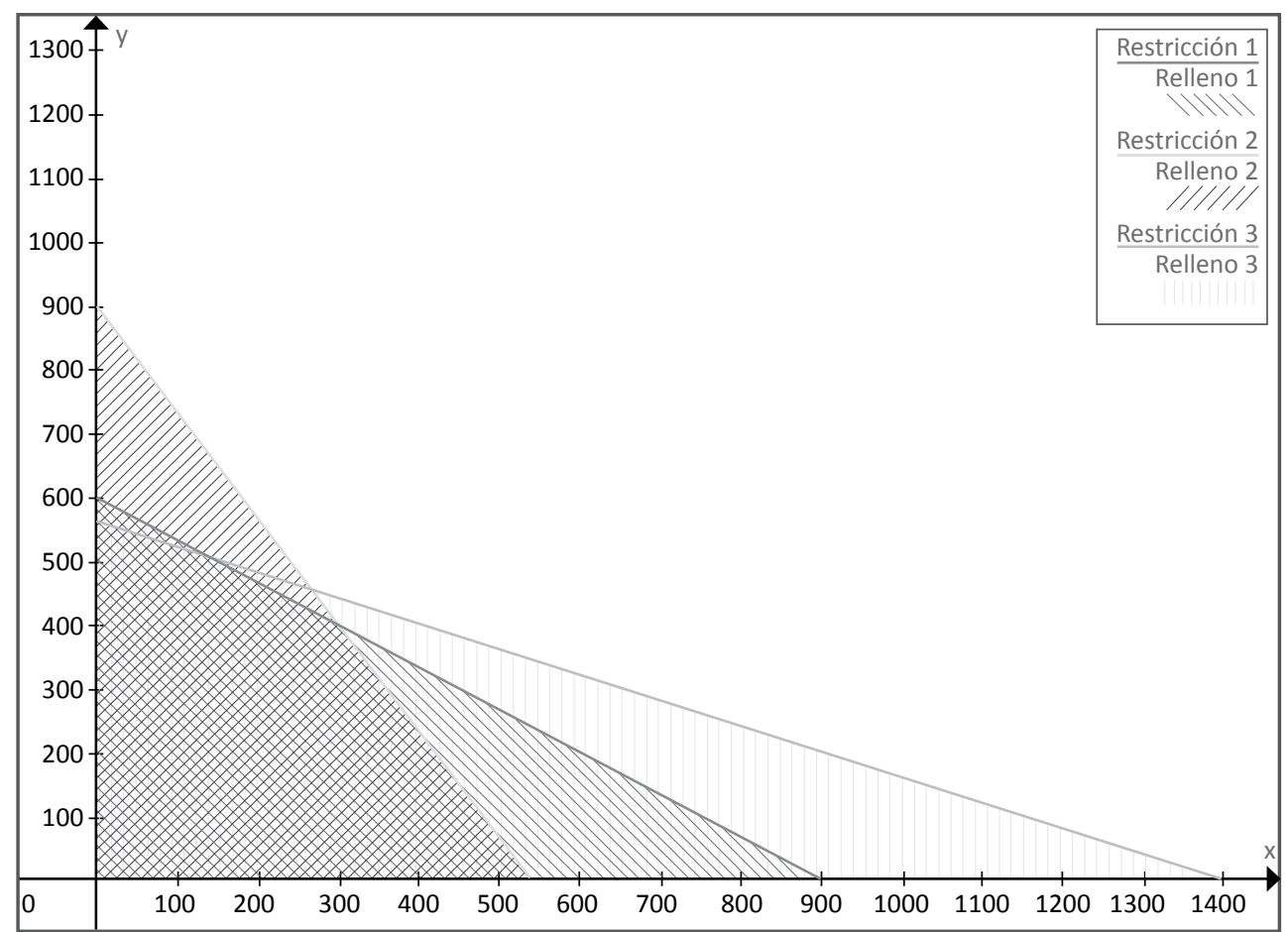

Gráfico 5. Área de soluciones factibles 
El área de soluciones factibles estará representada por el cuadriculado.

1.3) Una vez graficadas las restricciones podemos saber cuál es el o los puntos extremos ${ }^{5}$ que satisfacen el problema y permite obtener la máxima utilidad o beneficio bruto. ¿Cómo podemos saber cuáles son esos puntos con Graph?

En la parte izquierda de la pantalla de Graph, seleccionamos una de las restricciones y en la barra de menú elegimos Calcular y luego, Evaluar:

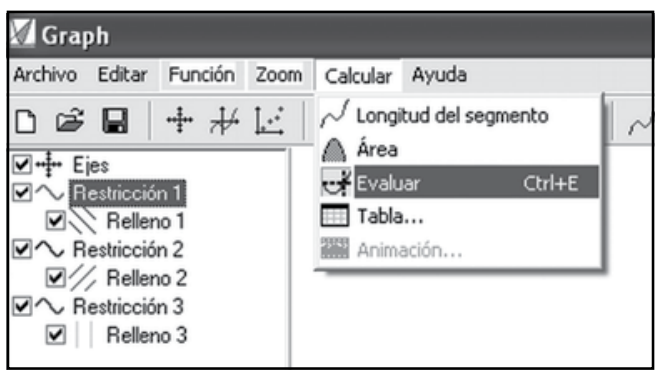

Gráfico 6. Calcular-Evaluar

Se activará una herramienta que nos permitirá movernos a lo largo de la función para verificar en qué punto se interceptan dos o más rectas.

Si prestamos atención a la parte inferior izquierda, podemos elegir la opción "Ver en: intersección”, luego, dando un solo clic en el gráfico, el programa nos mostrará las intersecciones.

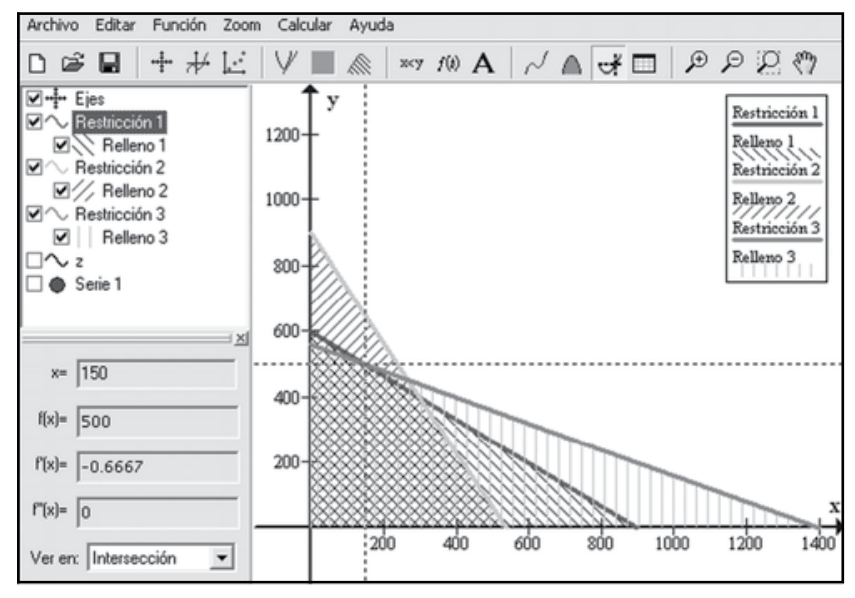

Gráfico 7. Intersección

La primera intersección se encuentra en $\mathrm{x}=150, \mathrm{y}=500$, es el punto (150; 500); la otra intersección está dada por los valores $\mathrm{x}=300, \mathrm{y}=400$ que corresponden al punto (300;400).

En este caso, el punto que indica la solución óptima es (150; 500). Decimos que es el punto óptimo porque es el que permite obtener el mayor valor de la función objetivo, lo que significa que en ese punto el beneficio es máximo.

\footnotetext{
${ }^{5}$ Puntos extremos: Son los que aparecen en la intersección de 2 líneas de frontera o en la intersección de una línea de frontera y un eje.
} 
1.4) $\mathrm{Al}$ conocer el punto óptimo podemos representar la función objetivo, recordemos que el punto óptimo pertenece a la misma:

$$
\begin{gathered}
z=5 x+8 y \\
z=5 * 150+8 * 500 \\
4750=5 x+8 y
\end{gathered}
$$

Se despeja “y”

$$
y=\frac{2375}{4}-\frac{5}{8} x
$$

Insertamos la función con un intervalo de o a 950.

1.5) Si se quiere resaltar el punto de la solución óptima:

* En la barra de Menú elegimos Función-Insertar serie de puntos y escribimos

$$
\mathrm{x}=150 ; \mathrm{y}=500
$$

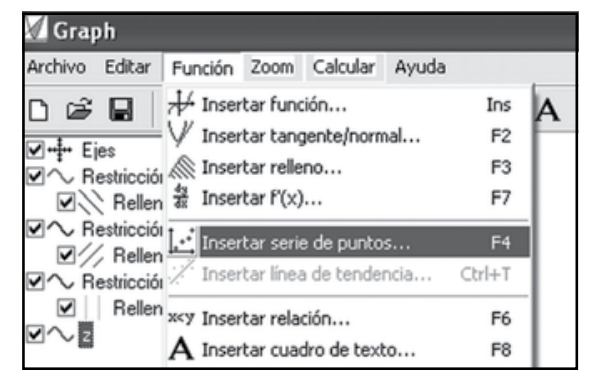

Gráfico 8. Función-Insertar serie de puntos

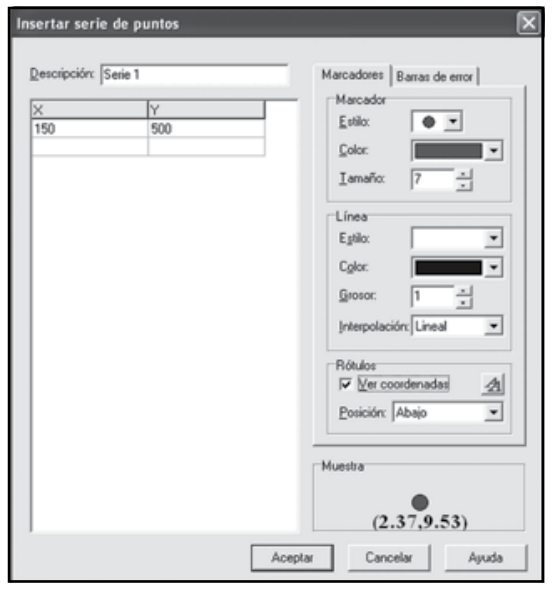

Gráfico 9. Insertar serie de puntos 
Como resultado final tenemos:

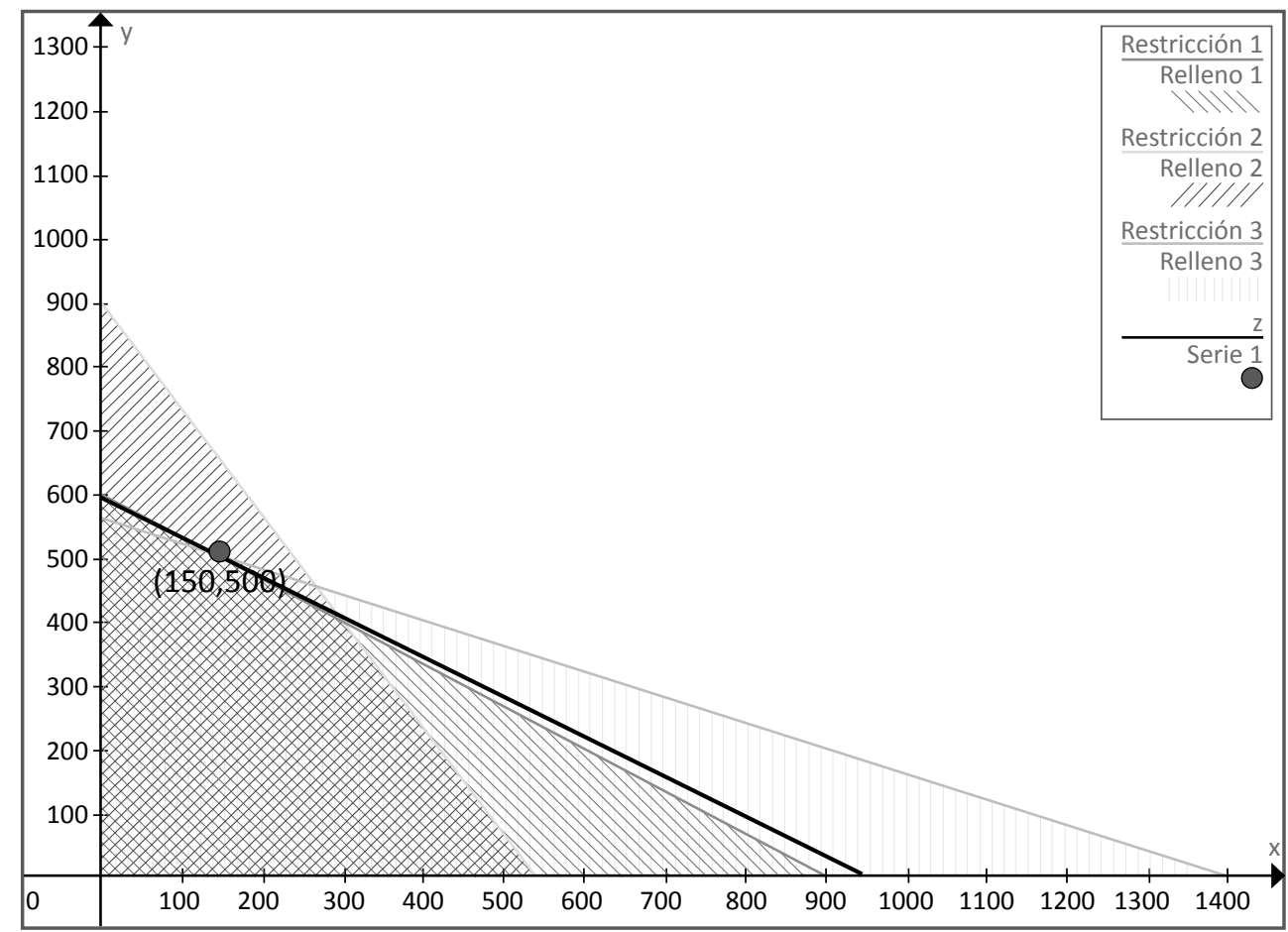

Gráfico 10. Resultado final

\section{HOJA DE CÁLCULO EXCEL Y SOLUCIÓN CON SOLVER}

Para distribuir los datos en la hoja de cálculo de Excel debemos distinguir lo siguiente:

1. Datos de entrada: Todos los datos numéricos necesarios para calcular la función objetivo y las restricciones deben aparecer en la hoja de cálculo.

2. Celdas cambiantes: Los valores de estas celdas pueden cambiarse para optimizar el objetivo.

3. Celda (función) objetivo: La celda objetivo contiene la función objetivo. La herramienta Solver varía los valores de las celdas cambiantes para optimizar el valor de la función objetivo.

$\mathrm{Al}$ definir la función objetivo en la celda $\mathrm{B} 4$ escribimos:

$$
=5^{*} \mathbf{B} 7+8 * \mathbf{B 8}
$$

Esta sumatoria de los productos parciales de las cantidades de cada una de los tipos de pelotas a producir por su correspondiente ganancia, es el valor óptimo.

4. Restricciones: En Excel, las restricciones no se muestran directamente en la hoja de cálculo, se especifican al abrir el menú de la aplicación Solver. Previamente, definimos las celdas que calculan el consumo de los distintos materiales, para establecer las restricciones 
más fácilmente. Este paso no es necesario, pero nos permite tener a la vista todos los datos y estar preparados para responder a preguntas como ¿cuántas horas de Corte y Costura fueron utilizadas?. Para contestarla, calculamos la cantidad del recurso utilizado para cada una de los tipos de pelotas. Para la primera restricción escribimos en la celda B12:

$$
=\mathrm{B}_{7}+\mathrm{B8}^{*} \mathbf{3} / \mathbf{2}
$$

Esto es, la suma de los productos parciales del recurso "Hs Corte y Costura" que se utiliza para la producción de las pelotas por la cantidad a fabricarse de cada uno de los tipos de pelotas.

Para las restricciones restantes debemos ingresar: $=\mathbf{1} / \mathbf{2}^{*} \mathbf{B} 7+\mathbf{3} / \mathbf{1 0}{ }^{*} \mathbf{B 8}$

$$
=\mathbf{1} / \mathbf{1 0}{ }^{*} \mathrm{~B} 7+\mathbf{1} / \mathbf{4}^{*} \mathrm{B8}
$$

Los datos volcados en la planilla Excel deben reflejarse con la siguiente imagen:

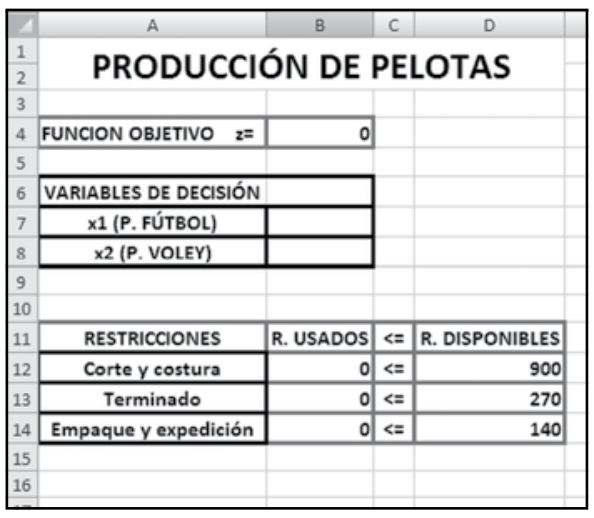

Gráfico 11. Distribución de datos en la hoja de cálculo

Completados los pasos anteriores, podemos introducir el modelo en el menú Solver.

El complemento Solver de EXCEL sirve para resolver problemas de optimización lineal y no lineal; también se pueden indicar restricciones enteras sobre las variables de decisión. Con Solver es posible resolver problemas que tengan hasta 200 variables de decisión, 100 restricciones explícitas y 400 simples (cotas superior e inferior o restricciones enteras sobre las variables de decisión). ${ }^{6}$

En el menú Datos, pestaña "Análisis" encontraremos el complemento Solver y al dar un clic sobre él, aparecerá un cuadro como el siguiente:

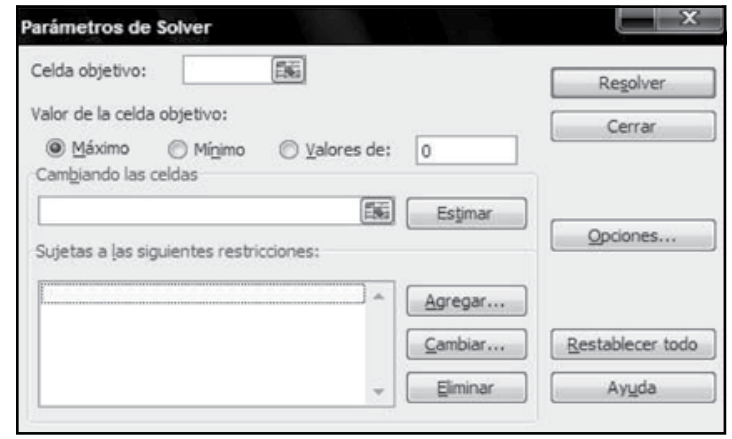

Gráfico 12. Parámetros de Solver 
Ingresamos el conjunto de restricciones con el botón “Agregar...”:

\begin{tabular}{|c|c|c|c|c|c|}
\hline \multicolumn{6}{|c|}{ Agregar restricción } \\
\hline \multicolumn{4}{|c|}{ Referencia de la celda: } & \multicolumn{2}{|l|}{ Restricción: } \\
\hline$\$ B \$ 12: \$ B \$ 14$ & 腎 & $<=$ & 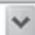 & $=\$ D \$ 12: \$ D \$ 14$ & 国國 \\
\hline Aceptar & Cancel & & & Agregar & Ayuda \\
\hline
\end{tabular}

Gráfico 13. Agregar restricción

Esto significa que la celda B12 debe ser menor o igual a D12, la celda B13, menor o igual a D13 y la celda B14 menor o igual a D14; es decir:

\section{Recursos usados $\leq$ Recursos disponibles para la elaboración}

Luego, en Parámetros de Solver, en la opción Celda objetivo elegiremos la celda B4 teniendo en cuenta que el Valor de la Celda objetivo debe ser maximizado.

Por último, la opción Cambiando celdas corresponde a las celdas vacías que Solver rellenará con la solución del problema: $\$ \mathrm{~B} \$ 7$ : $\$ \mathrm{~B} \$ 8$

Las restricciones de no negatividad indican que para que exista beneficio las variables de decisión deben son positivas y que si todas ellas fueran nulas no existe beneficio alguno. Para continuar resolviendo el modelo lineal seleccionamos el submenú Opciones de Solver y marcamos Asumir no negativos y Adoptar modelo lineal.

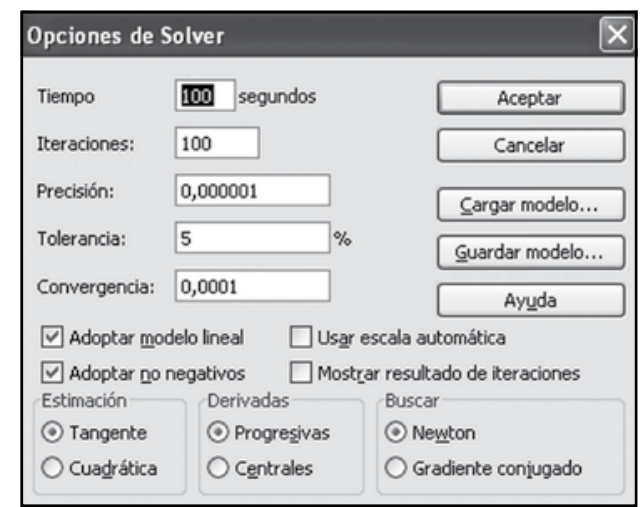

Gráfico 14. Opciones de Solver

En términos generales, los valores por defecto de la mayoría de estos parámetros son perfectamente válidos. ${ }^{7}$

Damos clic en "Resolver" y Solver tratará de buscar la solución óptima para el modelo planteado, de encontrarlo, aparece en pantalla un cuadro como el siguiente: 


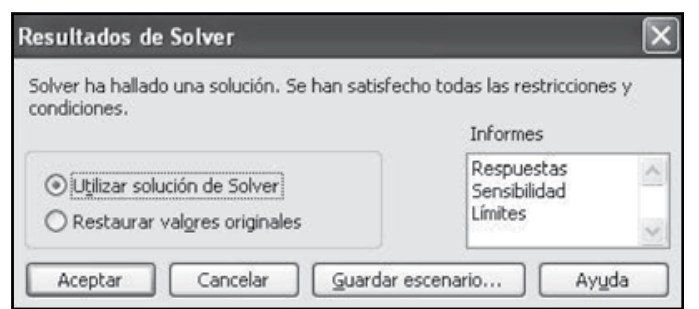

Gráfico 15. Resultados de Solver

Solver nos avisa que la solución ha sido hallada y debemos elegir si queremos utilizar esa solución o queremos volver a la planilla anterior restaurando los valores originales. También tenemos la posibilidad de elegir qué tipo de informes queremos que Solver nos brinde: Informe de Respuestas, Sensibilidad y/o Límites.

El informe se crea en una nueva hoja de cálculo del libro. Si Solver no encuentra una solución, la opción de crear un informe no está disponible.

El resultado que nos brinda Solver es el siguiente:

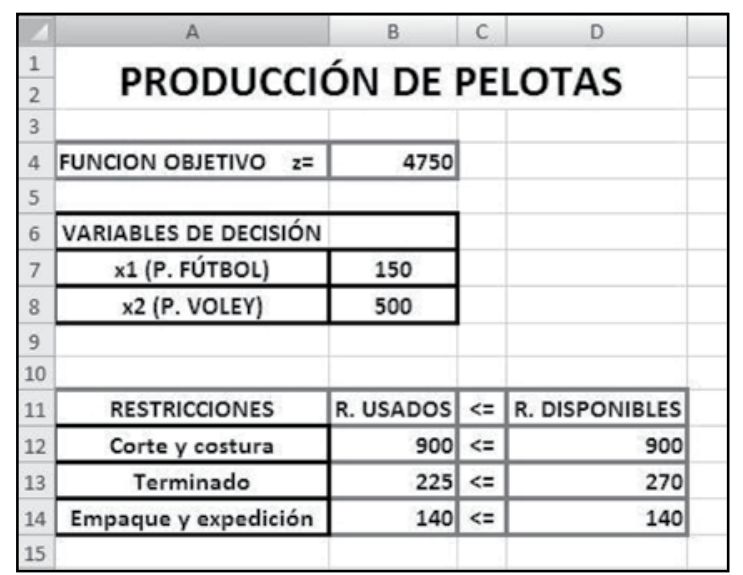

Gráfico 16. Resultados a través del Solver

Se puede ver que la solución óptima encontrada a través de Solver es la misma que se puede hallar a través de la solución gráfica del problema.

\section{ANÁLISIS DE SENSIBILIDAD O POST-ÓPTIMO}

Es el estudio de cómo los posibles cambios en los coeficientes de un problema de programación lineal afecta a la solución óptima.

Este análisis proporciona la información necesaria para responder a esos cambios sin requerir una solución completa del problema de programación lineal revisado o modificado. 


\section{INFORMES DE SOLVER}

Solver genera tres informes para problemas lineales: Informe de Respuestas, Informe de Sensibilidad y el Informe de Limites. En particular, el que nos brinda información sobre el análisis post-óptimo es el informe de sensibilidad.

\section{EL INFORME DE RESPUESTAS}

Nos muestra el valor inicial y final de cada celda de destino y de todas las celdas ajustables así como una lista de cada restricción y su estado (Opcional u Obligatorio).

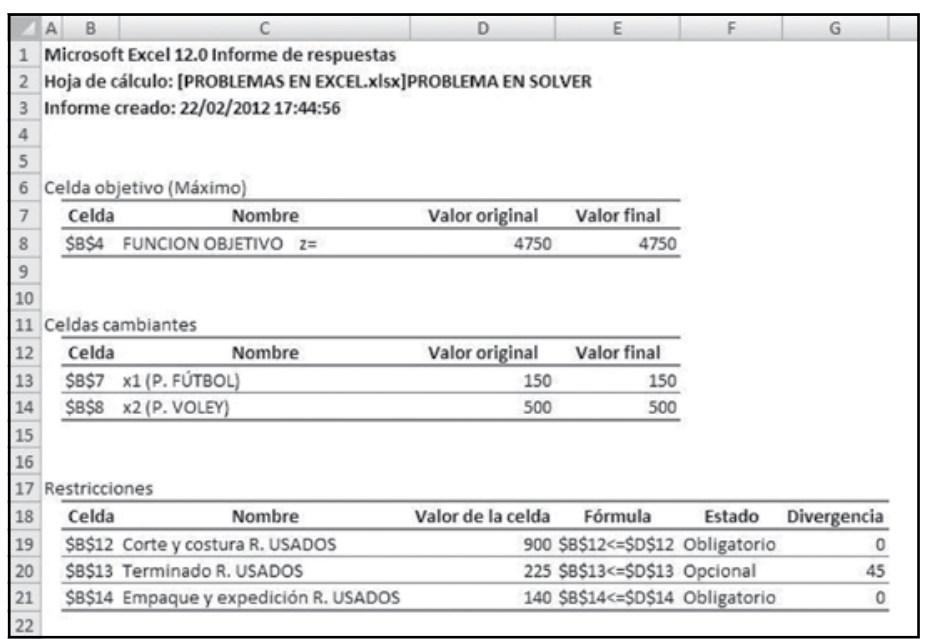

Gráfico 17. Informe de respuestas

En Celda Objetivo aparece la dirección de la optimización (Máximo), la celda de la función objetivo, el nombre, el valor original antes de optimizar y el valor óptimo (valor final).

En Celdas Cambiantes aparecen las celdas de las variables controlables o de decisión, el nombre, los valores iniciales de las variables y la solución óptima (valor final).

En Restricciones se observa:

* Valor de la celda: es el valor que toma el lado izquierdo de cada restricción en la solución óptima.

* Fórmula: nos recuerda las restricciones que hemos introducido, incluyendo si es de $\leq$, $=0 \geq \mathrm{y}$ las celdas referidas.

* Estado: Nos indica si la restricción se cumple exactamente, con una igualdad, y no hay un margen. En otras palabras, nos indica si la restricción es activa (obligatoria).

* Divergencia, Slack o Variables de Holgura: es el margen que tiene cada restricción. Si la desigualdad es $\leq$, entonces es el lado derecho de la restricción (la constante) menos el lado izquierdo. Si la desigualdad es $\geq$, es el lado izquierdo menos el lado derecho (la constante). Si la restricción es activa, desde luego el margen será cero. Indica las cantidades de 
los recursos no utilizados en el plan óptimo. Por lo tanto, podemos decir que los recursos que limitaron la producción fueron las Horas de Corte y Costura y Horas de Empaque y Expedición (no nos sobra horas para seguir produciendo pelotas) mientras que las horas disponibles de Terminado no fueron limitantes.

\section{EL INFORME DE SENSIBILIDAD}

Proporciona el valor óptimo de cada celda ajustable, su coste reducido, el coeficiente de función objetivo y el aumento y la disminución de éste para el cual la solución actual permanece óptima (el resto permanece fijo).

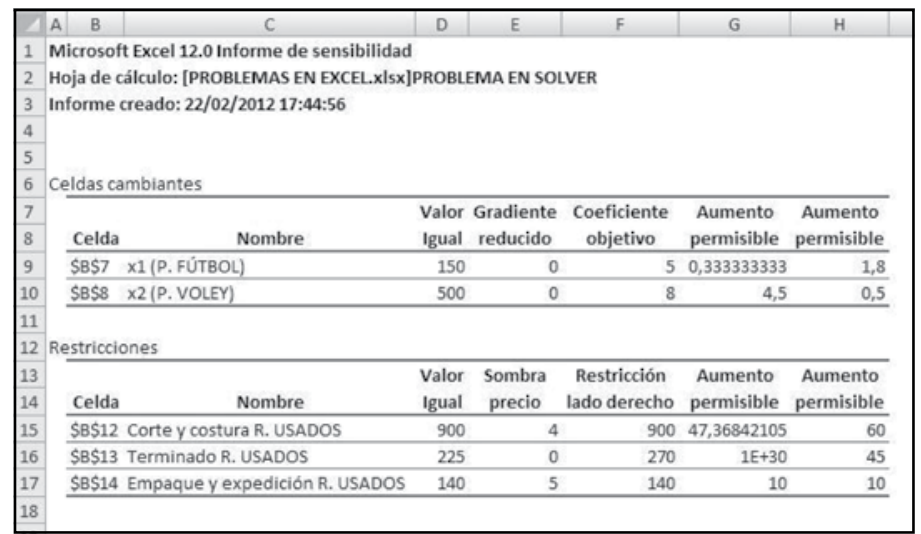

Gráfico 18. Informe de sensibilidad

\section{Celdas Cambiantes:}

* Valor Igual: son los valores óptimos de las variables de decisión.

* Costo reducido o Gradiente reducido: solo es significativa para las variables de decisión cuyo valor óptimo es igual a cero. El costo reducido de cualquier variable de decisión en particular se define como cuanto tendría que cambiar el coeficiente de dicha variable en la función objetivo para tener un valor optimo positivo. Por tanto, si una variable ya es positiva en el óptimo, su costo reducido es cero.

* Coeficiente objetivo: son los coeficientes de la función objetivo.

* Aumento permisible: incremento admisible en los coeficientes de la función objetivo sin que cambien los valores óptimos de las variables de decisión.

* Disminución permisible: disminución admisible en los coeficientes de la función objetivo sin que cambien los valores óptimos de las variables controlables.

Las columnas de "Aumento permisible" y "Disminución permisible”, indican el rango en que pueden modificarse los coeficientes objetivos de las variables sin que ello altere a la solución propuesta para las variables de decisión (sí alteraría el valor final del la F.O., salvo que el valor de la variable cuyo coeficiente que se modifico continúe siendo o). Hay que tener en 
cuenta que el rango es un intervalo abierto en las que estas disminuciones y aumentos no son válidos en el valor exacto que muestran en los extremos.

En el Informe de Sensibilidad de nuestro ejemplo, podemos ver que el precio de la Pelota de Fútbol puede disminuir hasta \$3,21 y la solución permanecería óptima (es decir, se seguiría produciendo lo mismo), y el precio de la Pelota de Voley puede aumentar hasta \$12,49 y, aun así, la solución permanecería óptima.

\section{CAMBIOS EN LA FUNCIÓN OBJETIVO}

La solución óptima de un modelo no cambia si un coeficiente objetivo de alguna variable en la función objetiva cambia dentro de cierto rango. Por ahora, solo se permite el cambio de un coeficiente a la vez. ¿Qué pasaría con la solución óptima si el coeficiente objetivo de la Pelota de Vóley cambia de $\$ 8$ a $\$ 9$ ? Dado que el coeficiente objetivo modificado cae dentro del intervalo en el que se acepta que puede aumentar (puede aumentar hasta $8+4,50=\$ 12,50$ ), se puede asegurar que no habrá cambios en la solución optima, es decir en las cantidades a producir.

\section{Restricciones}

* Valor igual: es el valor que toma el lado izquierdo de cada restricción en la solución óptima.

* Sombra Precio o Precio Dual: son la solución del problema dual. Son los precios duales, o precios sombra de los recursos (o requerimientos) indicados en las restricciones. Aquí, indican la mejora en el valor de la función objetivo si se "relaja" una desigualdad, o el empeoramiento si se la restringe. Nos muestra cual sería el aumento en la función objetivo si la variable aumentara en una unidad. Si se agregara una hora de corte y costura, la función objetivo experimentaría un crecimiento de $\$ 4$. Se conoce en economía con el nombre de producto marginal del recurso y este indica cuanto estaría el empresario dispuesto a pagar (máximo) por una unidad adicional de recurso limitante.

Suministra información relacionada con la productividad del recurso que se añada. Así por ejemplo una unidad adicional de horas corte y costura agregaría \$4 al valor de la función objetivo pero una unidad menos la reduciría en $\$ 4$, sin embargo un hora más del departamento terminado no agregaría nada dado que el recurso no se utilizó totalmente.

Los precios sombra de los recursos limitantes son distintos de cero, sin embargo de las no limitantes son iguales a cero.

Algunos textos hacen una diferencia entre los precios duales y precios sombra.

Los precios duales indican cuanto estaría el empresario dispuesto a pagar (máximo) por una unidad adicional de recurso limitante y los precios sombra el aumento o disminución de la función objetivo por el incremento de la constante.

Los precios sombra y duales son los mismos en los problemas de maximización, mientras que en los problemas de minimización, los precios sombra tienen signo negativo.

* Restricción lado derecho: indican los lados derechos de las desigualdades. 
* Aumento permisible: El Aumento Permisible representa en cuanto puede incrementarse el lado derecho (constante) sin que se altere el precio dual. Por ej. se puede incrementar el número de Hs Empaque y Expedición mejorando en \$5 por cada unidad de hora de trabajo del departamento empaque y expedición. Sin embargo esto es válido para un incremento de hasta 10 horas. Si disponemos más de eso, el precio dual será otro ya que aparecerán los rendimientos decrecientes.

* Disminución permisible: indica en cuánto puede disminuir el lado derecho de la restricción sin que cambie el precio dual de un recurso (o requerimiento).

El precio sombra de cada restricción (el cambio en la función objetivo por unidad de aumento en el lado derecho) se da junto con el aumento y disminución del valor del lado derecho para el cual es válido el precio. Por ejemplo, una disminución de 5 hs de Empaque y Expedición (está permitido una disminución de hasta 10 hs, un aumento mayor derivará en una situación en la que tendremos holgura de Hs Empaque y Expedición) dará como resultado un cambio en la función objetiva de $(-5)^{*}(5)=-25$ pesos. Lo mismo se aplica para cuando se aumenta la utilización del insumo, si se aumenta su utilización en 6 hs (está permitido una disminución de hasta 10, luego de ello el precio sombra variará) la función objetiva aumentará en \$30.

\section{CAMBIOS SIMULTÁNEOS}

En algunos casos podemos estar interesados en lo que sucede si dos lados o más se modifican simultáneamente. Es posible el análisis de cambios simultáneos con la ayuda de la regla de 100\%.

REGLA DEL 100\%:

Para todos los lados derechos que se cambian, sume los porcentajes de los aumentos permisibles y las disminuciones permisibles. Si la suma de los porcentajes es menor o igual que 100\%, los precios duales no cambian (Anderson, 2004).

Por ej: Se decide un aumento de 6 horas en el departamento corte y costura, que tiene permitido un aumento de hasta 47,36 horas y un aumento de 2 horas en el departamento empaque y expedición, que tiene permitido un aumento de hasta 10 horas. El aumento determinado para el departamento corte y costura representa $6 / 47,36 * 100=12,67 \%$ y el aumento determinado del departamento empaque y expedición representa $2 / 10 * 100=20 \%$. La suma de los porcentajes es $12,67 \%+20 \%=32,67 \%$, por lo tanto podemos decir que los precios duales no cambiarán. 


\section{INFORME DE LÍMITES}

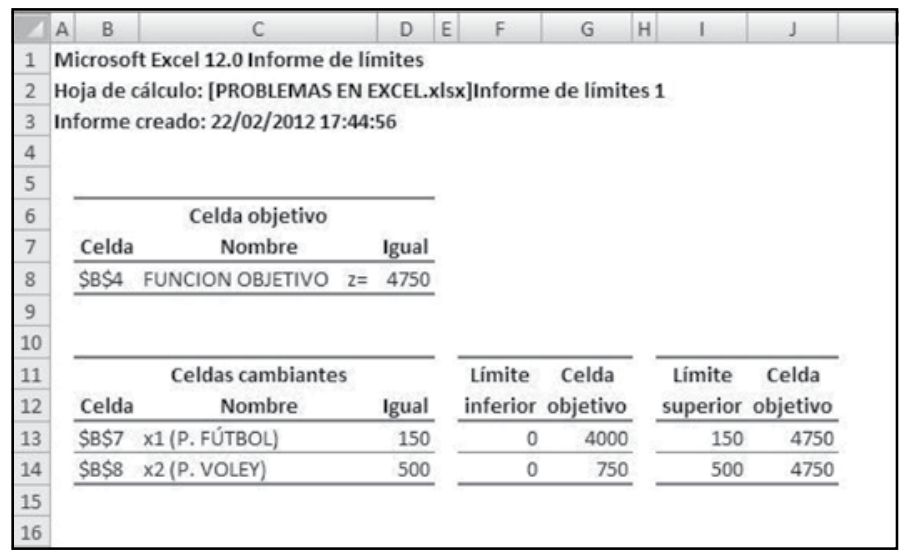

Gráfico 19. Informes de límites

Fue diseñado por Microsoft con el fin de suministrar un análisis diferente de sensibilidad.

Los especialistas suelen dar muchísima importancia a los informes de Respuesta y Sensibilidad, ya que ellos le permiten visualizar que pasaría si se cambian determinados parámetros.

El informe de límites muestra el rango de los valores que pueden asumir las celdas cambiantes (variables de decisión), basados en los restricciones que se hayan definido.

\section{Celda Objetivo:}

* Igual: Es la solución óptima encontrada: 150 unidades de pelotas de fútbol y 500 unidades de pelotas de vóley.

\section{Celdas Cambiantes}

* Límite Inferior: Es el menor valor que puede tomar la variable (suponiendo que las demás mantienen el valor óptimo encontrado), y satisfacer todas las restricciones.

* Límite superior: es el mayor valor que puede tomar la variable (suponiendo que las demás mantienen el valor óptimo encontrado) sin violar las restricciones.

* Celda Objetivo: Es el valor que toma la función objetivo si la variable considerada toma el valor del límite inferior y las demás variables mantienen el valor óptimo encontrado. Por ejemplo, cual es el valor de la celda objetivo suponiendo que el de la producción de las pelotas de fútbol es o: $\$ 4000$.

Ya estamos en condiciones de responder las preguntas que formulamos párrafos más arriba:

2. El máximo beneficio obtenible es de $\$ 4750$ a este nivel de producción.

3. La cantidad de pelotas de fútbol 5 a fabricarse es de 150 y la cantidad de pelotas de vóley, 500.

4. Las horas a programarse en el departamento "Empaque y Expedición” son de 140.

5. Existe holgura de un recurso, las horas del departamento "Terminado" ya que de 270 horas disponibles sólo se utilizaron 225 horas. La holgura es de 45 horas. En el resto de los departamentos la holgura es cero ya que se utilizaron totalmente las horas disponibles. 
6. Se puede ver que la solución óptima encontrada a través de Solver es la misma que se puede hallar a través del gráfico.

7. La restricción que no limita a la región factible en el punto de la solución óptima es la restricción 2, correspondiente a Hs Terminado. Esto quiere decir que se puede esperar que exista holgura en este recurso y de hecho, la holgura que se halló a través del Solver es de 45 horas.

Con este ejemplo mostramos el conjunto de estrategias puestas en juego para facilitar y optimizar el proceso de enseñanza-aprendizaje en el aula universitaria, convirtiendo las TIC en recursos y medios para mejorar la educación superior, adaptándonos a las nuevas generaciones y potenciando el valor agregado que conlleva su utilización.

\section{CONCLUSIONES}

Las TIC, como recurso didáctico nos permitieron lograr resultados que muestran el aporte del valor agregado del uso de ellas en el proceso de enseñanza-aprendizaje de contenidos matemáticos. El beneficio de la innovación realizada puede observarse en los datos que exponemos a continuación que son los resultados de los dos parciales de la asignatura en el ciclo lectivo 2011.

\begin{tabular}{|c|c|c|c|c|}
\hline & A Rendir & Presentes & Aprobados & Aprob/Pres \% \\
\hline Totales & 925 & 705 & 226 & $32,06 \%$ \\
\hline
\end{tabular}

Tabla 2. Datos del primer parcial

Debe tenerse en cuenta que el segundo parcial se toma luego de desarrollarse las unidades: 5-Matrices y Determinantes; 6-Sistemas de Ecuaciones y 7-Programación Lineal, unidad ésta última que encierra conceptos de las dos anteriores.

\begin{tabular}{|c|c|c|c|c|}
\hline & A RENDIR & PRESENTES & APROBADOS & Aprob/Pres \% \\
\hline Totales & 933 & 654 & 464 & $70,95 \%$ \\
\hline
\end{tabular}

Tabla 3. Datos del segundo parcial

Estos cuadros reflejan lo satisfactorio de la innovación realizada, habiendo pasado de un $32,06 \%$ de aprobados sobre los presentes en el primer examen a un 70,95\% de los presentes en el segundo examen.

\begin{tabular}{|c|c|c|}
\hline & REGULARIZADOS & \% Reg/A Rendir \\
\hline ANTES DEL RECUPERATORIO & 210 & $22,51 \%$ \\
\hline DESPÚES DEL RECUPERATORIO & 402 & $43,09 \%$ \\
\hline
\end{tabular}

Tabla 4. Datos del recuperatorio

Esto permitió que la gran mayoría de los alumnos pudieran rendir satisfactoriamente el único recuperatorio de parciales que tienen, lo que incrementó el número de regulares de la asignatura.

Sin ser taxativa, podemos dar la siguiente lista de resultados obtenidos: 
* Adquisición de nuevos conocimientos, tanto de matemática como de informática.

* Posibilidades de investigación, porque el aprendizaje del soft permitió que los alumnos investigaran sobre otras operaciones que pueden realizarse con el mismo.

* Posibilidad de identificar, seleccionar, guardar, organizar y analizar los datos disponibles en cualquier momento.

* Suplemento virtual que permite en la observación de gráficos, iteraciones y movimientos, formar una imagen del camino al valor óptimo.

* Comprensión de análisis post-óptimo.

* Trazado de estrategias para minimizar situaciones de error.

* Contribución a la formación de recursos humanos.

* Conversión del alumno en sujeto activo del proceso educativo, con la correspondiente satisfacción de los mismos al comprobar sus capacidades para realizar cálculos complejos en forma precisa y con economía de tiempo.

* Mayor disposición de los alumnos al aprendizaje ante la posibilidad de utilizar herramientas informáticas.

* Mejoramiento de la infraestructura de las instituciones educativas.

* Aumento del abanico y de la calidad de los trabajos de Matemática.

Esta experiencia en el manejo de las TIC nos lleva a considerar la posibilidad de aplicarlas en todos los contenidos del programa de Matemática I que se desarrollan con gráficos y cálculos, para el mejor aprovechamiento de estos recursos. Posiblemente en el ciclo lectivo del año 2013 todos los temas sean tratados con éstas herramientas informáticas, lo que potenciará la calidad de nuestro trabajo.

\section{REFERENCIAS BIBLIOGRÁFICAS}

Besil, A.C. (2007). Economía, manual introductorio $5^{\text {a }}$ edición. Corrientes: Universidad Nacional del Nordeste. Editorial Universitaria de la Universidad Nacional del Nordeste. Chaco. Argentina.

Córica, J.L. y Dinerstein, P. (2009) Diseño Curricular y Nuevas Generaciones: Incorporando a la generación NET. $1^{\circ}$ edición. E.V.A. Mendoza. Argentina.

Ferreiro, R. (2000). Nuevos ambientes de aprendizaje. En Córica y Dinerstein (2009). Diseño Curricular y Nuevas Generaciones: Incorporando a la generación NET. $1^{0}$ edición. E.V.A. Mendoza. Argentina.

Anderson, D.; Sweenney, D.; Williams, T. (2004). Métodos cuantitativos para los negocios. $9^{\text {a }}$ Edición. International Thompson Editores. U.S.A. 


\section{CURRÍCULUM VITAE}

RESCALA, CARMEN.

Profesora en Matemática, Física y Cosmografía, Contadora Pública y Especialista en Ingeniería Gerencial. Actualmente es Profesora Titular Ordinaria Dedicación Exclusiva de la Cátedra "Matemática I" de la Facultad Ciencias Económicas de la UNNE y Profesora Titular Ordinaria Dedicación Simple de la Cátedra" Ciencias Básicas" de la Facultad de Arquitectura y Urbanismo de la UNNE. Miembro Fundador y Miembro del Consejo Académico del Instituto de Matemática de la Facultad de Ingeniería de la UNNE. Integrante de la Comisión Directiva en el cargo de Pro-secretaria de la Asociación Nacional de Docentes de Matemática de Facultades de Ciencias Económicas y Afines. Es Docente Investigadora, Categoría 3.

carmenrescala@yahoo.com.ar

\section{ROHDE, GRICELA ALICIA.}

Profesora en Matemática y Cosmografía y Especialista en Investigación Educativa. Actualmente es Profesora Adjunta Ordinaria con Dedicación Exclusiva de la Cátedra "Matemática I" y Auxiliar Docente de Primera de la Cátedra "Matemática II" de la Facultad de Ciencias Económicas de la UNNE. Es Docente Investigadora, Categoría 4.

\section{grohde@eco.unne.edu.ar}

\section{PAVÓN, RITA ALEJANDRA.}

Alumna de $4^{\circ}$ año de la carrera contador público. Becaria de Pre-Grado s/ Res. No970/11 e integrante del grupo de investigación en el proyecto Moo1/2010 "Modelos Matemáticos y Estadísticos para la Gestión de Administración de Empresas Constructoras (Segunda Etapa)”.

Los autores integran el Proyecto acreditado por la Secretaría General de Ciencia y Técnica de la UNNE MOO1/2O1O, cuyo título es: MODELOS MATEMÁTICOS Y ESTADÍSTICOS PARA LA GESTIÓN DE ADMINISTRACIÓN DE EMPRESAS CONSTRUCTORAS (Segunda Etapa), siendo Directora del mismo la Prof. Carmen Rescala y Co-Director, el Ing. Gustavo Devicenzi. 